\title{
Peritoneal Necrosis, CTCAE
}

National Cancer Institute

\section{Source}

National Cancer Institute. Peritoneal Necrosis, CT CAE. NCI Thesaurus. Code C57850.

A disorder characterized by a necrotic process occurring in the peritoneum. 